\title{
Phagocytic function of tumor-associated macrophages as a key determinant of tumor progression control: a review
}

\author{
Marc Lecoultre, ${ }^{1,2}$ Valérie Dutoit,, ${ }^{1,2,3}$ Paul R Walker ${ }^{1,2}$
}

To cite: Lecoultre M, Dutoit V, Walker PR. Phagocytic function of tumor-associated macrophages as a key determinant of tumor progression control: a review. Journal for ImmunoTherapy of Cancer 2020;8:e001408. doi:10.1136/jitc-2020-001408

Accepted 15 November 2020

Check for updates

(c) Author(s) (or their employer(s)) 2020. Re-use permitted under CC BY-NC. No commercial re-use. See rights and permissions. Published by BMJ.

${ }^{1}$ Faculty of Medicine, University of Geneva, Geneva, Switzerland ${ }^{2}$ Center for Translational Research in Onco-Hematology, University of Geneva, Geneva, Switzerland

${ }^{3}$ Faculty of Medicine, Laboratory of Tumor Immunology and Center of Oncology, Geneva University Hospital, Geneva, Switzerland

Correspondence to

Dr Paul R Walker;

Paul.Walker@unige.ch

\section{ABSTRACT}

Tumor-associated macrophage (TAM) phagocytic activity is emerging as a new mechanism to harness for cancer treatment. Currently, many approaches are investigated at the preclinical level and some modalities have now reached clinical trials, including the targeting of the phagocytosis inhibitor CD47. The rationale for increasing TAM phagocytic activity is to improve innate anticancer immunity, and to promote T-cell mediated adaptive immune responses. In this context, a clear understanding of the impact of TAM phagocytosis on both innate and adaptive immunity is critical. Indeed, uncertainties persist regarding the capacity of TAM to present tumor antigens to CD8 T cells by cross-presentation. This process is critical for an optimal cytotoxic T-cell immune response and can be mediated by dendritic cells but also potentially by macrophages. In addition, the engulfment of cancer cells affects TAM functionality, as apoptotic cell uptake (a process termed efferocytosis) promotes macrophage anti-inflammatory functions. Because of the abundance of TAM in most solid tumors and the common use of apoptosis inducers such as radiotherapy to treat patients with cancer, efferocytosis potentially affects the overall immune balance within the tumor microenvironment (TME). In this review, we will discuss how cancer cell phagocytosis by TAM impacts antitumor immunity. First, we will focus on the potential of the phagocytic activity of TAM per se to control tumor progression. Second, we will examine the potential of TAM to act as antigen presenting cells for tumor specific CD8 T cells, considering the different characteristics of this process in the tumor tissue and at the molecular level. Finally, we will see how phagocytosis and efferocytosis affect TAM functionality and how these mechanisms impact on antitumor immunity. A better understanding of these aspects will enable us to better predict and interpret the consequences of cancer therapies on the immune status of the TME. Future cancer treatment regimens can thereby be designed to not only impact directly on cancer cells, but also to favorably modulate TAM phagocytic activity to benefit from the potential of this central immune player to achieve more potent therapeutic efficacy.

\section{INTRODUCTION}

Tumor-associated macrophage (TAM) are an abundant part of the immune infiltrate in most solid tumors. ${ }^{2}$ They can derive both from blood monocytes attracted by chemokines such as CCL2 or CSF-1, and from tissue-resident macrophages. ${ }^{3}$ The tumor microenvironment
(TME) impacts TAM functionality and promotes a wound healing-like response, which in the context of cancer actively promotes tumor growth. The interactions between tumor and TAM and their roles in tumor growth have already been discussed in excellent reviews. ${ }^{3-7}$ The most explored and described mechanisms encompass TAM secretion of growth factors, promotion of tumor-associated angiogenesis, and induction of an immunosuppressive or anti-inflammatory microenvironment. To achieve this, TAM secrete different antiinflammatory cytokines, such as Transforming growth factor (TGF) $\beta$ and interleukin (IL)10, express different immune checkpoint ligands including programmed death-ligand 1 (PD-L1), and starve cytotoxic CD8 T cells by depleting essential amino acids through arginase expression. In addition, TAM recruit regulatory $\mathrm{T}$ cells (Treg) that participate in antitumor immune response inhibition. ${ }^{4}$ In this context, TAM accumulation correlates with an unfavorable prognosis in many cancer types, such as melanoma, breast, pancreatic, ovarian, head and neck, bladder and renal cell cancer. ${ }^{8}$ Notwithstanding the evidence for these protumor characteristics, the plasticity of macrophages and their potential to phagocytose cancer cells raise the interesting possibility that TAM could indeed manifest antitumor activities if appropriately re-educated. ${ }^{5}$ Indeed, a significant body of work has been undertaken to understand how TAM phagocytic activity and antigen presentation might contribute to TAM antitumor characteristics.

In this review, we will discuss the impact of cancer cell phagocytosis by TAM on antitumor immunity. We will focus on the potential of this phagocytic activity of TAM per se to control tumor progression, on the potential of TAM to act as antigen presenting cells (APC) for tumor-specific CD8 T-cell activation (through cross-presentation), and on how phagocytosis affects TAM functionality. We will discuss the existing evidence regarding 
these different mechanisms and the unresolved issues that should be addressed.

\section{TAM SHARE CERTAIN CHARACTERISTICS WITH DENDRITIC CELLS}

The functional and phenotypical similarities between TAM and dendritic cells (DC) force us to interpret the literature on TAM with caution. Indeed, TAM share their phagocytic proprieties with the other cells of the mononuclear phagocyte system present in solid tumors: DC and monocytes. ${ }^{9}$ Although monocytes do not have a strong phagocytic activity, they are precursors of TAM and monocyte-derived DC. It is beyond the scope of this review to present in detail the functions of the different DC subsets; these have already been addressed in other reviews. ${ }^{10}$ Nevertheless, it is important to stress that DC, and mainly classical type 1 DC (cDC1), have a central role in T-cell induction against tumors by presenting antigens and secreting IL-12, a key cytokine promoting CD4 Th1 and CD8 T-cell activity, ${ }^{11}$ and that a specific depletion of these cells prevents CD8 T-cell mediated immunity. ${ }^{12}$ Importantly, DC have a well-documented capacity to cross-present tumor antigens to CD8 T cells. ${ }^{13}$ In addition to TAM, monocytes and DC, another mononuclear immune cell population was reported in the TME: the monocytic myeloid-derived suppressor cell (M-MDSC). ${ }^{14}$ The phenotype and functions of M-MDSC greatly overlap with TAM and monocytes. Consequently, it is not always clear whether published findings can be attributed to M-MDSC or to TAM. We therefore do not specifically discuss M-MDSC in this review, but we remain open to the possibility that future research will address whether the different mechanisms described herein also apply to this cell type.

In tumors, where DC and TAM share many similarities regarding their origins (both partially deriving from monocytes), as well as their tissue localization and their functions, it is critical to define them accurately. Different cell markers are expressed in mice and human DC and TAM (figure 1). In mice, TAM is usually defined by $\mathrm{CD} 11 \mathrm{~b}$ and $\mathrm{F} 4 / 80$, while $\mathrm{DC}$ are identified by expression of CD11c. However, it was shown that some DC also express F4/80, ${ }^{15}$ while CD11c has been detected on macrophages in vitro. ${ }^{16}$ More specific markers for macrophages exist, such as MerTK and CD64, ${ }^{17}$ but they are much less frequently used than CD11b and F4/80. In humans, the distinction between macrophages and DC is made with relatively specific markers such as CD68 for macrophages and BDCA1 and BDCA3 for CDC2 and cDC1, respectively. However, it was noted that, in breast cancer, CD68 expression was not fully specific for TAM. ${ }^{18}$ Whether these findings also apply to other tumors has not been reported. Overall, since phenotypic markers used to characterize TAM and DC do not always perfectly discriminate between these lineages, our interpretation of functional data must take this into account.

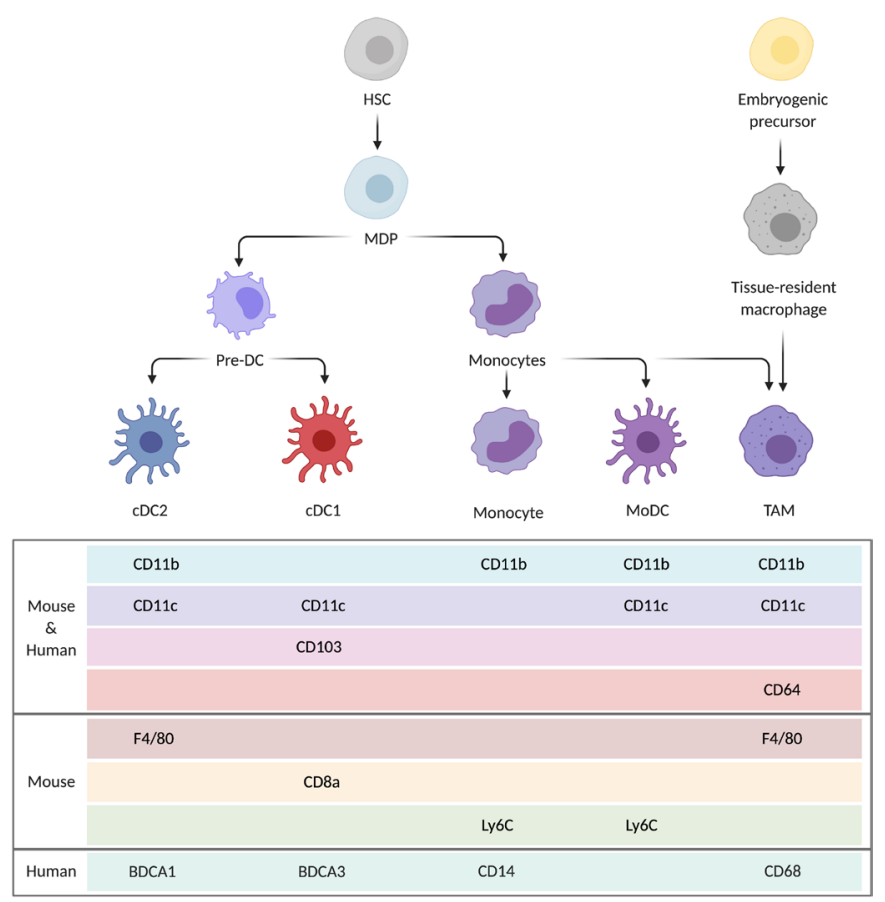

Figure 1 Mononuclear phagocytes present in tumors and common markers used to distinguish them. HSC give rise to the different subclasses of DC (CDC1, cDC2), monocytes and monocyte-derived DC (MoDC). This common origin causes an overlap regarding the different markers they express. TAM derive from both HSC via monocytes and from tissue-resident macrophages originating from embryogenic precursors. cDC, classical dendritic cells; DC, dendritic cells; HSC, hematopoietic stem cell; MDP, macrophage DC progenitor; MoDC, monocyte-derived DC; TAM, tumorassociated macrophages.

\section{IS TAM PHAGOCYTIC ACTIVITY ALONE ABLE TO CONTROL TUMOR PROGRESSION? \\ Balance between "eat-me" and "don't eat-me" signals orchestrates phagocytosis initiation}

A central mechanism by which TAM can affect cancer progression is through phagocytosis of tumor cells. Most eukaryotic cells can engulf small particles by endocytosis, but only professional phagocytes, including macrophages and DC, uptake particles bigger than $\sim 0.5 \mu \mathrm{m}$ by phagocytosis. ${ }^{19}$ Molecular mechanisms of phagocytosis have been reviewed in detail elsewhere ${ }^{20}$; in this review, we focus on the factors modulating phagocytic activity and its impact on tumor control.

Interactions between tumor cells and TAM that regulate phagocytosis are the result of "eat-me" ligands (eg, calreticulin, SLAMF7, opsonizing antibodies, phosphatidylserine (PtdSer)) and "don't eat-me" ligands (eg, CD47, PD-L1, major histocompatibility complex (MHC) I) ${ }^{21}$ expressed on the surface of tumor cells, which bind to specific receptors on macrophages (see figure 2 for details). The balance between these different functional classes of molecules exposed on cancer cells dictates initiation of phagocytosis. If "eat-me" signals prevail, they induce the rearrangement of the actin cytoskeleton in phagocytes, driving cell engulfment. Among the "don't 


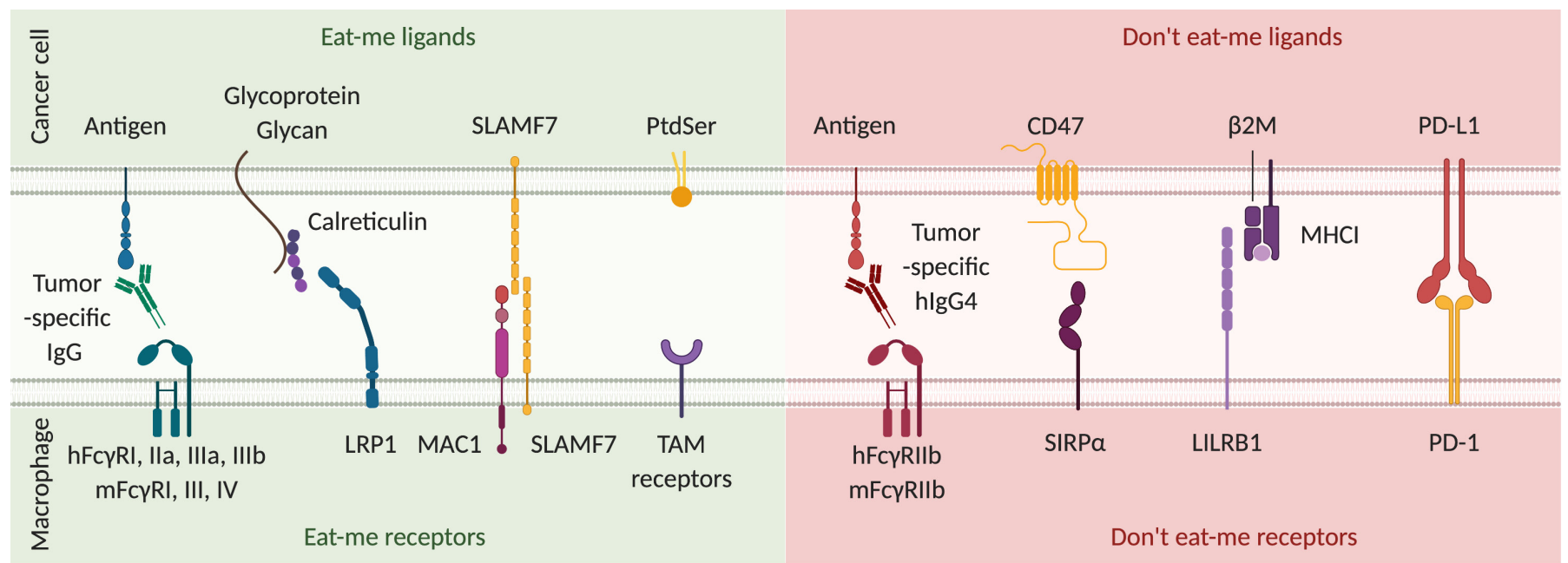

Figure 2 Signals modulating phagocytosis initiation. From left to right: tumor-specific IgG antibodies opsonize cancer cells by

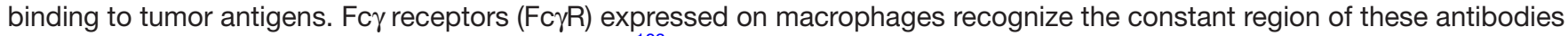
and initiate antibody-dependent cell phagocytosis. ${ }^{103}$ In humans, the intracellular portion of FcyRI, Ila, Illa and IIlb possess an immunoreceptor tyrosine-based activation motif (ITAM) that leads to pro-phagocytic activity. In mice, FcyRI, III and IV possess an ITAM. Calreticulin translocation to the surface is induced by cellular stress and DNA damage. ${ }^{104}$ Once on the surface of cancer cells, it is stabilized by glycoproteins and glycans and binds to the lipoprotein receptor-related protein 1 (LRP1) on phagocytes. The exact mechanism of action of signaling lymphocytic activation molecule family member 7 (SLAMF7) is unclear, but it promotes cytoskeletal reorganization required for phagocytosis ${ }^{105}$ through interaction with macrophage antigen 1 (MAC1) on phagocytes. Phosphatidylserine (PtdSer) is specifically expressed by apoptotic cells and binds to many different receptors among which those of the tumor-associated macrophage (TAM) family (TYRO3, AXL, MerTK) are the best described. It induces efferocytosis, which is a phagocytic process specific for the uptake of apoptotic cells. Eat-me receptor activity is counterbalanced by don't eat me receptors. First, in both humans and mice, FcyRllb possesses an immunoreceptor tyrosinebased inhibition motif (ITIM) that negatively regulates initiation of phagocytosis. In humans, IgG4 binds with the highest affinity

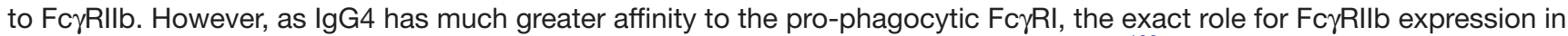
vivo is still unknown. In mice, IgG1, IgG2a and IgG2b all bind with low affinity to FcyRllb. ${ }^{103}$ CD47 is the most important "don't eat me" ligand. SIRP $\alpha$, its receptor on macrophages, inhibits myosin II polymerization, which is a critical step in initiation of cell engulfment. Major histocompatibility complex class I (MHC I) expression by cancer cells also confers protection against phagocytosis. Leukocyte immunoglobulin-like receptor 1 (LILRB1) binding to the $\beta 2$-microglobulin component of MHC I prevents phagocytosis. ${ }^{106}$ Finally, programmed cell death protein 1 (PD-1) expression on macrophages is correlated with a lower phagocytic activity, which is restored in PD-1 deficient macrophages. Thus, PD-1 activation by its ligand PD-L1 expressed by cancer cells is another inhibitory signal for phagocytosis. ${ }^{35}$ Overall, the balance of signaling through eat-me and don't eat-me receptors will determine initiation of the phagocytic process.

eat-me" ligands, CD47 binding to its receptor signalregulatory protein $\alpha$ (SIRP $\alpha)$ on macrophages inhibits myosin II conformational changes and thereby inhibits phagosome formation. ${ }^{22}$ Inhibition of CD47 or SIRP $\alpha$ with antagonistic antibodies increases the phagocytic activity of TAM and decreases tumor growth in various preclinical models such as glioblastoma, ${ }^{23}$ melanoma, ${ }^{24}$ lymphoma,${ }^{25}$ breast $^{26}$ and colorectal cancer. ${ }^{27}$ Encouraged by these promising results, more than a dozen of phase I clinical trials are ongoing. ${ }^{28}$ However, it is still unknown whether TAM alone are sufficient to control tumor growth or whether an involvement of $\mathrm{T}$ cells is required, as we will now discuss.

\section{Is phagocytic activity of TAM sufficient to control tumor growth?}

The use of antagonistic anti-CD47 antibody to control growth of human tumors xenografted in NOD $/ \mathrm{SCID} / \gamma$ (NSG) mice lacking T cells was efficacious for medulloblastoma and pediatric glioblastoma, ${ }^{23}$ adult glioblastoma, ${ }^{29}$ as well as ovarian, bladder, colorectal and breast tumors. ${ }^{30}$
These different studies confirmed the effect in immunocompetent mice, without, however, formally excluding the involvement of T cells. Using an antagonistic SIRP $\alpha$ antibody in the Raji lymphoma model, ${ }^{25}$ a synergy with rituximab was observed, resulting in increased phagocytosis, highlighting the key role of antibody-dependent cell phagocytosis (ADCP) in the context of CD47-SIRP $\alpha$ inhibition. Similar involvement of ADCP was reported in the context of direct CD47 blockade. ${ }^{31}$ Of note, in the aforementioned xenograft models, antihuman anti-CD47 antibody was used, enabling administration of high antibody doses without on-target off-tumor toxicity. The latter would be expected in a syngeneic setting because of ubiquitous CD47 expression by normal murine cells, such as erythrocytes. Xenograft studies therefore potentially over-estimate the efficacy of this approach. To limit the impact of anti-CD47 on healthy tissues, Dheilly et al have developed bispecific antibodies, one arm targeting CD47 and the other a tumor specific antigen. ${ }^{32}$ In patients, this approach should provide a better specificity of anti-CD47 
for cancer cells and reduce side effects. Besides CD47 inhibition, other strategies increasing TAM phagocytic activity have been tested. In an immunocompetent model of pancreatic ductal adenocarcinoma (PDAC), the tolllike receptor (TLR) 9 agonist CpG impacted TAM lipid metabolism and thereby their membrane deformity, resulting in increased phagocytic activity and inhibition of tumor growth. ${ }^{33}$ Consistent with a CpG-mediated promotion of macrophage phagocytosis, the effect was conserved in T-cell deficient Rag2 ${ }^{-/-}$mice and after DC depletion using CD11c-DTR mice. Overall, these studies suggest that, in certain mouse models, the phagocytic activity of TAM alone is sufficient to eradicate tumor cells, or at least inhibit tumor growth. In humans, CD47 expression by cancer cells is correlated with poor prognosis, ${ }^{30}$ suggesting that constitutive phagocytosis might impact immunosurveillance. However, direct quantification of phagocytic activity in patient samples is not feasible using the techniques used in mice (principally quantification of fluorescently labeled cancer cell uptake by macrophages). Therefore, no definitive evidence exists regarding TAM capacity to phagocytose cancer cells without anti-CD47 or other phagocytosis inducers in patients.

The obligatory involvement of $\mathrm{T}$ cells in the context of CD47-SIRP $\alpha$ inhibition has been shown in other syngeneic models. In the MPA/DMBA-induced breast cancer model, the positive impact of anti-CD47 on survival was abolished when CD4 and CD8 T cells were depleted. ${ }^{26}$ In B16F10 melanoma, inhibition of CD47 was efficient only when associated with PD-L1 targeting, suggesting that both an increase of phagocytosis by TAM and reinvigoration of $\mathrm{T}$ cells are required for optimal tumor control. ${ }^{34}$ However, this interpretation has to be considered with caution, since PD-L1 binding to programmed cell death protein 1 on macrophages also inhibits phagocytosis, ${ }^{35}$ potentially ruling out the need for $\mathrm{T}$ cells in this antiCD47/anti-PD-L1 combination. Nevertheless, another study using the same melanoma model demonstrated synergistic effects between anti-CD47 and CTLA-4. ${ }^{36}$ As CTLA-4 is not a regulator of phagocytosis, this confirms that, in melanoma, efficient antitumor immunity requires not only TAM phagocytic activity, but also T cells. Finally, in a fibrosarcoma model, efficient control of tumor growth by combining irradiation and $\mathrm{CD} 47$ blockade was CD8 T cell-dependent. ${ }^{37}$

Overall, while the increase of TAM phagocytic activity in T-cell deficient NSG mice was sufficient to control tumor growth, a T-cell dependency was observed in some immunocompetent models, highlighting the importance of understanding the interactions between these cell populations. Indeed, TAM can favor T-cell responses by not only secreting proinflammatory cytokines, but also by presenting tumor antigens on MHC I and II to CD8 and CD4 T cells, respectively. ${ }^{38}$ It is established that macrophages efficiently present engulfed exogenous antigenic material on MHC II, ${ }^{39}$ while antigen presentation on MHC I was initially described for endogenous cytosolic antigens in every nucleated cell. However, APC have the ability to load engulfed antigens on MHC I through a mechanism called cross-presentation. This latter pathway is critical for CD8 T-cell mediated antitumor immunity, but it is not clearly established whether it is only promoted by DC or whether TAM also play a role.

\section{ARE TAM ABLE TO EFFICIENTLY CROSS-PRESENT TUMOR ANTIGENS TO CD8 T CELLS? Evidence for cross-presentation by TAM}

The importance of cross presentation for CD8 T-mediated immunity in cancer was shown by Huang et $a l^{40}$ but without identifying the specific role of DC or TAM. In recent years, the balance of evidence points to DC as being the most efficient cross-presenting cells in vivo (as reviewed in Cruz et $a l^{41}$ ), although macrophages also possess cross-presentation potential. A key role of cross presentation is to prime naïve $\mathrm{T}$ cells, which takes place principally in the draining lymph node (LN) ${ }^{42}$ While DC migration and CCR7-mediated LN homing is well documented, the limited migration capacity of TAM and their low CCR7 expression prevent their trafficking to draining LN. ${ }^{10}$ Thus, the activity of TAM as APC takes place exclusively in the TME, where the evidence of naïve T-cell priming is controversial. In LN-deficient mice implanted with different tumors, T-cell activation in the TME was observed, butappeared to be TAM independent, ${ }^{43}$ whereas another study showed the capacity of $\mathrm{CD} 11 \mathrm{c}^{+} \mathrm{F} 4 / 80^{+}$cells to prime naive $\mathrm{T}$ cells in the TME. ${ }^{44}$ Tertiary lymphoid structures play a key role for naive T-cell priming directly in the TME, ${ }^{45}$ but the participation of TAM in this process has not been described. In this context, the role of TAM as APC in the TME seems to be primarily for T-cell reactivation, and any capacity to prime naïve $\mathrm{T}$ cells remains to be documented. For immunotherapy, the importance of T-cell reactivation in the TME was suggested in the context of adoptive T-cell transfer, where tumor specific T-cell retention ${ }^{46}$ and proliferation ${ }^{47}$ in situ were dependent on cross-presentation, without, however, an exploration of the exact involvement of DC or TAM. Recently, the importance of reactivating $\mathrm{T}$ cells in the TME has been highlighted by the demonstration that tissue-resident memory $\mathrm{T}$ cells are important for robust antitumor immunity. ${ }^{48}$ Regarding TAM, several studies have shown that cross-presentation function can be revealed using different approaches, as will be discussed. Indeed, in view of the abundance of TAM in many solid tumors and the clinical opportunities to manipulate their function, it is timely to reconsider evidence for and against their involvement in this process.

Several studies have shown the importance of DC and the dispensable role of TAM in CD8 T-cell priming. In the immunogenic GL261-OVA GBM model, Malo et al showed that a tumor-specific T-cell response was dependent on DC but not on TAM. ${ }^{49}$ In mice with conditional MHC I knock-out (KO) in DC (under the CD11c promoter) or in macrophages (under the lysozyme M promoter), T-cell infiltration in tumors was abolished in DC MHC I 
KO mice after ovalbumin vaccination, but not in macrophage MHC I KO mice. An important study by Xu et al showed that anti-CD47 treatment of MC38 colon cancer promoted preferential cross-presentation by DC rather than by TAM, although the latter showed enhanced phagocytosis. ${ }^{50}$ The mechanism involved activation of the nicotinamide adenine dinucleotide phosphate (NADPH) oxidase NOX2 in the phagosome, thereby limiting phagosome acidification and facilitating cytosolic translocation of proteins and DNA. Cytosolic DNA activated the cyclic GMP-AMP synthase (cGAS)/stimulator of interferon genes (STING) pathway and promoted crosspresentation of tumor antigens by $\mathrm{CD} 11 \mathrm{c}^{+} \mathrm{DC}$. In a PDAC model, TAM activation promoted a proinflammatory response, but T-cell activation was cDC1 dependent. ${ }^{51}$ In one of the rare studies in patients addressing the role of TAM versus DC, moDC and TAM isolated from peritoneal ascites of patients with cancer were used as APC to present the Melan-A/MART1 melanoma antigen to CD8 $\mathrm{T}$ cells; only moDC could efficiently activate $\mathrm{T}$ cells by secretion of the proinflammatory cytokine IL-12, which was not secreted by macrophages. ${ }^{52}$

Unlike the above-mentioned studies excluding TAM cross-presentation, others have shown that, by manipulating TAM phagocytic activity, tumor-derived antigens were efficiently presented to CD8 T cells. Using a human colon cancer cell line transfected with ovalbumin, Tseng et al showed in vitro that anti-CD47 antibodies promoted phagocytosis by murine macrophages and presentation of the ovalbumin-derived peptide to MHC I restricted OT-1 T cells ${ }^{53}$; similar results were achieved with murine GBM cells in vitro. ${ }^{54}$ In other in vitro studies using human cells, Barrio et al showed that interferon (IFN) $\gamma$ activated human macrophages presented the Melan-A/MART-1 antigen to $\mathrm{T}$ cells, resulting in activation. ${ }^{55}$ In a fibrosarcoma model, Muraoka et al used a cholesteryl pullulan nanogel to target tumor antigen to TAM, which then induced proliferation of specific CD8 T cells ex-vivo. ${ }^{56}$ However, TAM were defined as $\mathrm{CD} 11 \mathrm{~b}^{+} \mathrm{F} 4 / 80^{+}$, which would also be compatible with cDC2. Recently, Klichinsky et al developed chimeric antigen receptor macrophages targeting the HER2 tumor antigen that efficiently phagocytosed cancer cells, cross-presented tumor antigens in vitro, and prolonged NSG mouse survival in lung metastasis and peritoneal carcinosis models, ${ }^{57}$ opening new perspectives for TAM manipulation in cancer therapeutics. These different observations, mostly in vitro, suggest that macrophages have the capacity to cross-present tumor antigens to CD8 $\mathrm{T}$ cells, when their phagocytic activity is appropriately enhanced.

Overall, macrophages have a constitutively lower capacity than DC to cross-present tumor antigens. However, since the cross-presenting machinery exists in macrophages, it is possible that in some conditions or after therapeutic targeting, they could play a role in enhancing T-cell responses.

\section{Molecular mechanisms of cross-presentation}

The details of the intracellular cross-presentation pathway have been mostly elucidated in vitro with DC. We will discuss the general features and highlight those that differ in macrophages (figure 3). A better understanding of the limited capacity of macrophages for antigen processing at the molecular level could provide new targets to unlock the cross-presentation capacity of TAM.

Cross-presentation of tumor antigens relies on the capacity of APC to engulf cancer cells or cancer cell debris, mainly by phagocytosis, then to process their protein content in order to generate peptides of $8-10$ residues in length that can be loaded on MHC I. The proteolytic activity of the phagosome is initiated rapidly after cell engulfment when its fuses with early endosomes, inducing acidification to $\mathrm{pH}$ 6.5. Then, the phagosome fuses with late endosomes and lysosomes to mature into a phagolysosome, a step that induces further acidification (to $\mathrm{pH} 5$ ), mainly due to the recruitment of the V-ATPase proton pump. ${ }^{20}$ This low $\mathrm{pH}$ promotes activity of proteolytic enzymes that start to cleave large proteins into smaller peptides. Besides low $\mathrm{pH}$, proteases are also activated through oxidation by the NADPH oxidase NOX2 located on the phagolysosome membrane. ${ }^{58}$ Importantly, proteolytic activity was shown to be higher in macrophages than in DC. ${ }^{59}$ This would be consistent with longer antigen persistence in DC, a feature that was detected particularly in cross-presenting DC subsets. ${ }^{60}$ Thus, higher proteolysis that reduces antigen persistence is the first antigen processing limitation that is encountered for efficient cross-presentation by macrophages.

Once proteolysis is initiated in the phagosome, peptides are processed to a length that is compatible with MHC I loading. Here, two different mechanisms have been described: the cytoplasmic and the vacuolar pathways, the former being dominant for cross-presentation during cancer immunosurveillance in vivo. ${ }^{61}$

The cytoplasmic pathway is characterized by the translocation of ingested and partially proteolyzed peptides from the phagolysosome to the cytoplasm. The endoplasmic reticulum (ER)-associated degradation (ERAD) machinery that normally facilitates retro-translocation of misfolded proteins from the ER lumen into the cytosol plays an important part in this process. Indeed, the ERAD proteins SEC61 and p97 are required for crosspresentation in DC, ${ }^{62}$ suggesting that ER components are recruited in the phagosome. In addition, the heat-shock protein HSP90 plays a central role by refolding proteins in the cytoplasm after their translocation through the phagosome membrane. ${ }^{63}$ Interestingly, cytosolic translocation appeared be less efficient in macrophages than in DC, ${ }^{64}$ providing a second mechanism limiting their cross-presentation capacity. In the cytoplasm, proteasome cleavage finalizing antigen processing was shown to be essential for cross-presentation by macrophages in in vitro studies. ${ }^{65}$ Proteasome-generated peptides then follow the classical MHC I pathway, generally through translocation to the ER lumen but also via retro-translocation 


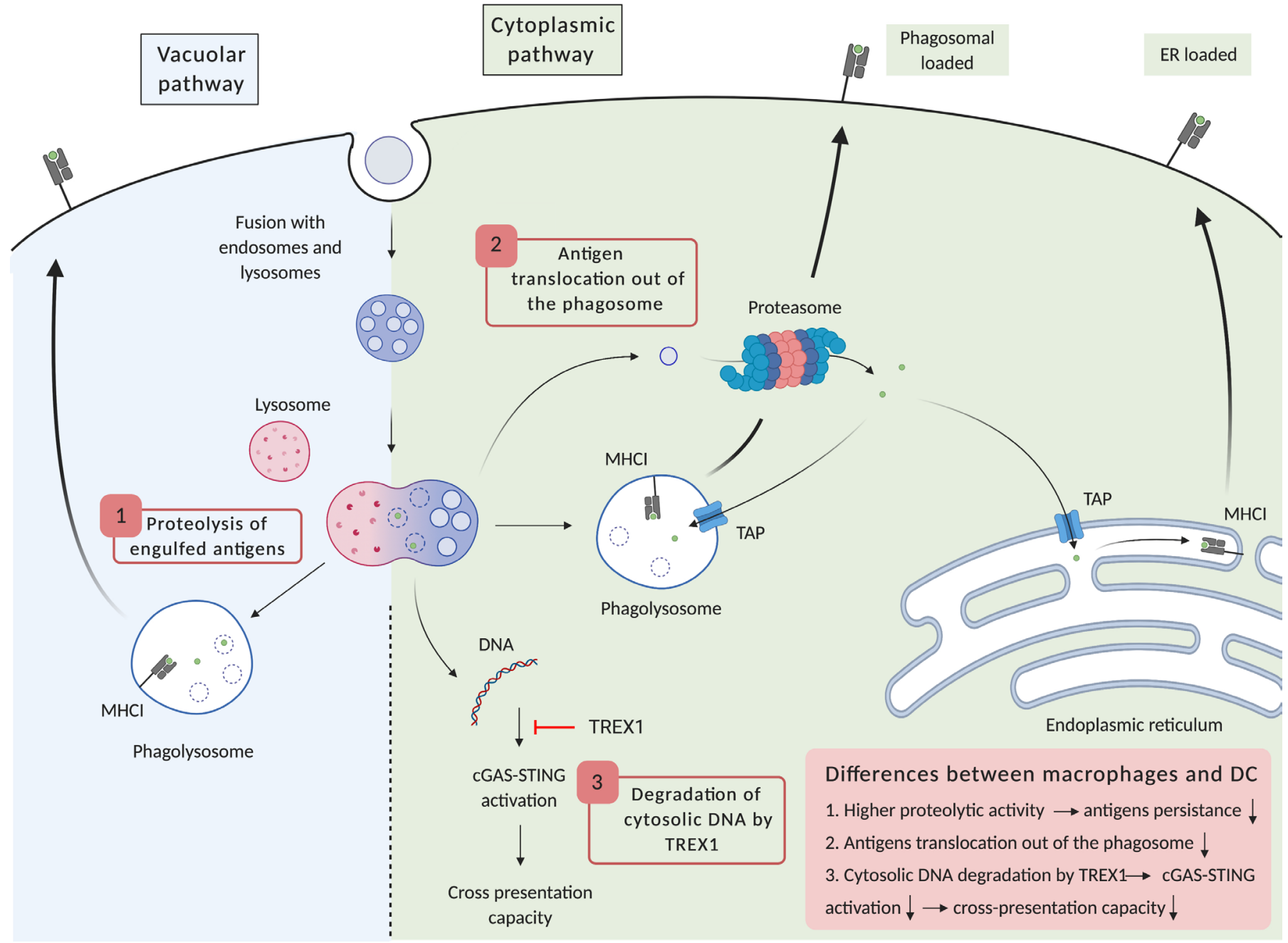

Figure 3 Differences in antigen processing reduce the capacity of macrophages for cross-presentation. Two different crosspresentation pathways have been described: the vacuolar pathway (left) and the cytoplasmic pathway (right). (1) Higher proteolytic activity in the macrophage phagolysosomes leads to a more rapid degradation of ingested antigens and limits their presentation on $\mathrm{MHCl}$. (2) Macrophage capacity to translocate antigens from the phagosome to the cytosol is lower than in DC. As this step is critical for the cytoplasmic pathway, it limits cross-presentation in macrophages. (3) Finally, expression of the cytosolic exonuclease TREX1 is increased in macrophages in response to external stimuli such as irradiation or TLR4 agonists. this limits CGAS-STING pathway activation and type I interferon secretion, which facilitate antigen persistence in phagolysosomes and cross-presentation. cGAS, cyclic GMP-AMP synthase; DC, dendritic cells; ER, endoplasmic reticulum; $\mathrm{MHCl}$, major histocompatibility complex I; STING, stimulator of interferon genes; TAP, transporter associated with antigen processing; TLR, toll-like receptor; TREX1, three-prime repair exonuclease 1.

in the phagosome, as the latter compartment was shown to possess the machinery required for MHC I-antigen complex generation. ${ }^{66}$ This step is dependent on the transporter associated with antigen processing (TAP) 1 and TAP2 proteins, as their loss of function prevents peptide loading on MHC I. ${ }^{65}$ Finally, once in these compartments, proteasome generated peptides are trimmed by amino-peptidases to be loaded on MHC I, ER-associated aminopeptidase 1 (ERAP1) in the $\mathrm{ER}^{67}$ and endosomal insulin-responsive aminopeptidase (IRAP) in the phagosome. ${ }^{68}$ Once loaded with antigens, MHC I molecules are translocated to the cell surface to be recognized by CD8 T cells.

Unlike the cytoplasmic pathway, the vacuolar pathway is resistant to proteasome and TAP inhibition. On the other hand, antigen presentation is prevented by cathepsin $\mathrm{S}$ inhibition, ${ }^{69}$ highlighting the key role of phagosomal proteolysis in this pathway. With the presence of MHC I in this compartment, this suggests that antigen processing and MHC I loading can both occur in the phagolysosome. Once antigenic peptide is bound, the MHC I-peptide complex can translocate directly to the cell surface.

In addition to protein antigens, DNA from phagocytosed cells can also escape into the cytosol where it activates the cGAS-STING pathway. ${ }^{70}$ cGAS is a cytosolic DNA sensor that catalyzes the formation of cyclic dinucleotide guanosine monophosphate-adenine monophosphate (cGAMP), which is recognized by the STING protein. Once activated, STING promotes the secretion of proinflammatory cytokines including type I IFNs 
and tumor necrosis factor (TNF)- $\alpha$ by DC and macrophages. ${ }^{71}$ Type I IFNs were shown to increase the $\mathrm{pH}$ of the phagolysosome of DC and to extend the persistence of ingested material, thus increasing the capacity of DC to cross-present. ${ }^{72}$ In T-cell mediated antitumor responses, cGAS-STING activation in DC is essential, as T-cell responses are abolished in STING-deficient mice, resulting in loss of tumor control. ${ }^{73}$ In this context, the activity of the cytosolic exonuclease three-prime repair exonuclease 1 (TREX1) induced by irradiation dampens activation of cGAS-STING by degrading cytosolic DNA, and potentially placates the immune response. ${ }^{74}$ Besides irradiation, TLR4 agonists increase TREX1 expression to a greater extent in macrophages than in DC. ${ }^{75}$ Interestingly, this study also showed that TREX1 limits upregulation of the costimulatory molecule CD86 in lipopolysaccharide-stimulated macrophages. Thus, increased degradation of cytosolic DNA in macrophages could be a third mechanism limiting their cross-presentation ability.

Overall, it appears that cross-presentation requires a low level of protein degradation in order to preserve antigens to be presented to CD8 T cells. With a higher proteolytic activity in the phagolysosome, antigen persistence in macrophages is reduced, thereby limiting cross-presentation. ${ }^{59}$ Furthermore, the translocation of antigen into the cytosol, a critical step for the cytoplasmic pathway, is less efficient in macrophages than in DC. ${ }^{64}$ Finally, the higher propensity of macrophages to increase TREX1 expression reduces the availability of cytosolic DNA for cGAS-STING activation, thereby limiting macrophage activation and capacity for efficient cross-presentation (figure 3 ).

The different studies presented earlier underline that constitutive cross-presentation by macrophages is less efficient than by DC, even though it can be enhanced using different approaches. The functional limitations of TAM might therefore be potentially counterbalanced by quantitative advantages. Indeed, in comparison with DC, TAM are generally more abundant ${ }^{9}$ and their phagocytic activity is generally higher. ${ }^{50}$ It would be of considerable interest to investigate whether the respective antigenpresenting roles of DC and TAM are related to the relative abundance of these APC at the tumor site. Indeed, this ranges from the relatively DC-rich TME of melanoma or lung cancer ${ }^{7677}$ to the DC-poor, but microglia-rich and TAM-rich TME of brain tumors, ${ }^{78} 79$ and we can therefore speculate that TAM may play a more important role in glioblastoma immune surveillance. Whether priming of naive T cells by TAM in the TME is an important phenomenon in vivo remains to be demonstrated; it is more probable that $\mathrm{T}$-cell reactivation occurs at this site, the former being a less stringent process. Thus, the capacity of TAM to cross-present tumor antigens could be a key mechanism for adoptive $\mathrm{T}$ cell transfer or when tissue resident memory $\mathrm{T}$ cells are harnessed for anticancer immunity.

\section{IMPACT OF EFFEROCYTOSIS AND PHAGOCYTOSIS ON TAM FUNCTIONS}

There is differential expression of "eat-me" ligands by cancer cells depending on whether they are apoptotic or necrotic. Indeed, caspase 3 cleavage promotes PtdSer exposure at the cell surface, which binds to specific receptors on macrophages, such as TYRO3, AXL, MerTK, TIM4 and Stabilin2 ${ }^{80}$ Engulfment of apoptotic cells after engagement of these receptors is called efferocytosis and, unlike phagocytosis, promotes tolerogenic functions of macrophages. In cancer, over $50 \%$ of patients are treated with radiotherapy, which is a strong inducer of apoptosis subsequent to DNA damage. ${ }^{81}$ Irradiation also increases CXCL12 and CSF-1 secretion by cancer cells that promote monocyte infiltration. ${ }^{81}$ This common treatment modality is likely to impact TAM functions and reshape the TME landscape.

As mentioned previously (see figure 2), uptake of cancer cells expressing "eat-me" signals associated with stress or necrosis promotes macrophage capacity to function as APC. ${ }^{21}$ In contrast, efferocytosis induces a tolerogenic response. Indeed, efferocytosis plays an important role during embryological development, negative selection of $\mathrm{T}$ cells, and clearance of apoptotic cells in the retina, ${ }^{82}$ where any unleashed inflammation could be catastrophic. The uptake of apoptotic neutrophils by macrophages has been particularly well studied; it promotes secretion of the anti-inflammatory cytokines IL-10 and TGF $\beta$ and decreases the secretion of proinflammatory IL-12. ${ }^{83}$ Binding of an apoptotic cell to MerTK counterbalances activation by TLR agonists and decreases secretion of type I IFNs (figure 4). ${ }^{85}$ In addition, it upregulates the E3 ubiquitin ligase suppressor of cytokine signaling (SOCS) 1 and SOCS3, thereby blocking signal transducer and activator of transcription 1 signaling and decreasing secretion of proinflammatory cytokines. ${ }^{86}$ The shifting of cytokine secretion towards those with anti-inflammatory functions impacts the TME by promoting the recruitment of other anti-inflammatory cells such as Tregs. ${ }^{87}$ Besides placating proinflammatory functions of macrophages, efferocytosis also restricts antigen presentation. Indeed, unlike phagosomes, efferosomes lack the machinery to load peptides onto MHC II for presentation to CD4 T cells. ${ }^{88}$ Instead, efferosomes recruit the recycling regulator RAB17 that enables ingested antigens to bypass MHC II processing. Regarding CD8 T cells, antigens can be exported from efferosomes and presented on MHC I, but because of the absence of costimulatory molecules, the result can be tolerogenic.$^{89}$ In addition, $\mathrm{pH}$ decrease in efferosomes is more rapid ${ }^{90}$ and more profound ${ }^{91}$ than in phagosomes, rapidly degrading antigenic material and limiting its persistence and availability for presentation to $\mathrm{T}$ cells. This is amplified by a block in TLR signaling by MerTK, limiting TLR-mediated promotion of antigen processing and presentation. Additional external factors can also facilitate efferocytosis initiation. IL-10 increases expression of MerTK in monocytes, ${ }^{92}$ potentiating a positive feedback loop between these two immunosuppressive 


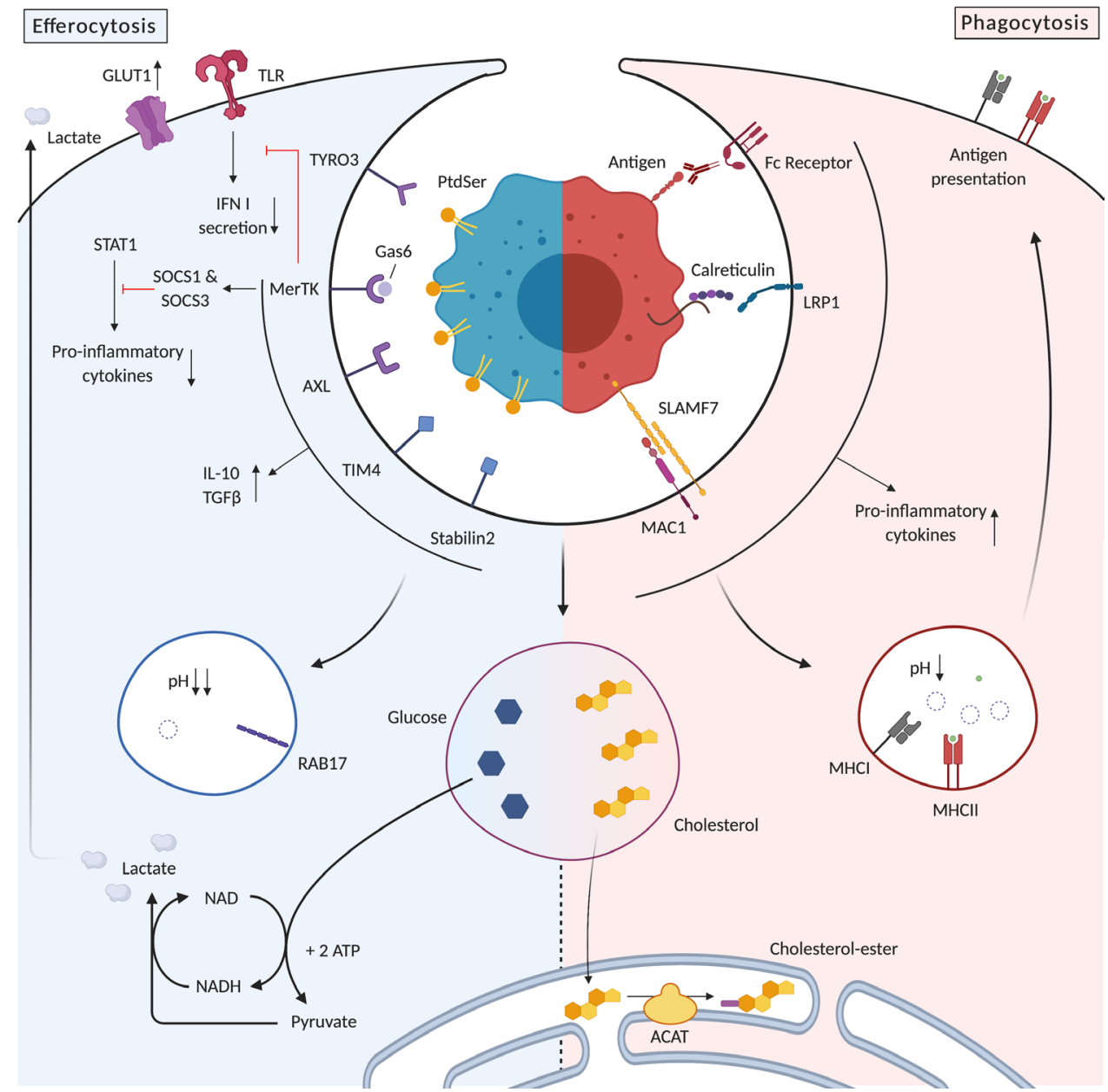

Figure 4 Efferocytosis promotes anti-inflammatory functions while phagocytosis promotes proinflammatory functions of tumor associated macrophages. Specific receptors for efferocytosis inhibit the downstream signaling of TLR, decrease proinflammatory cytokine secretion through signal transducer and activator of transcription (STAT) 1 inhibition and promote IL-10 and TGF $\beta$ secretion. Efferosomes are more acidic than phagosomes, thereby rapidly degrading antigens and limiting the cross-presentation capacity of phagocytes. In contrast, phagocytosis receptors promote an immunogenic response by secreting proinflammatory cytokines and promoting antigen presentation on $\mathrm{MHC}$ I and $\mathrm{MHC}$ II. Efferocytosis promotes aerobic glycolysis using glucose from engulfed cells but also from the environment through upregulation of the glucose transporter GLUT1. This metabolism contributes to the immunosuppressive tumor microenvironment by depleting glucose and secreting the glycolysis by-product lactate. Potentially cytotoxic quantities of cholesterol are transported to the endoplasmic reticulum where they are esterified by acyl-CoA:cholesterol acyltransferase (ACAT). IFN, interferon; IL, interleukin; LRP1, lipoprotein receptor-related protein 1; NADH, nicotinamide dinucleotide; MAC1, macrophage antigen1; MHC, major histocompatibility complex; SOCS, suppressor of cytokine signaling; SLAMF7, signaling lymphocytic activation molecule family member 7; TGF $\beta$, Transforming growth factor $\beta$; TLR, toll-like receptor.

mechanisms. Moreover, corticoids, potent immunosuppressors widely used in oncology for patient supportive care, were reported to increase MerTK expression in macrophages. $^{93}$

Targeting efferocytosis has been tested as an immunotherapy modality in the MC38 colon cancer model, ${ }^{94}$ where efferocytosis blockade by MerTK receptor inhibition led to secondary necrosis of cancer cells, favoring phagocytosis rather than efferocytosis. TAM secreted more IFN-1 $\beta$ and tumor control was achieved in a CD8 T-cell dependent manner. Thus, efferocytosis promotes an anti-inflammatory TME by impacting TAM functions directly, and by rapidly eliminating apoptotic cells that prevents damage-associated molecular pattern release associated with secondary necrosis.

Phagocytosis can also alter TAM functions through its impact on TAM metabolism ${ }^{795}$ (figure 4). Indeed, the engulfment of entire cells leads to an excess of lipids, cholesterol, proteins, carbohydrates and nucleic acids leading to metabolic stress for the phagocyte, regardless of the viability state of the cell engulfed. The important increase of cholesterol concentration is potentially cytotoxic for the phagocyte. This condition was studied in detail in atherosclerosis, where macrophages engulfed cholesterol-loaded apoptotic cells and became foam cells containing high quantities of 
cholesterol. Ingested cholesterol was delivered to the ER where acyl-CoA:cholesterol acyltransferase transformed it to cholesterol-ester to limit its cytotoxic effect. ${ }^{96}$ This metabolic surcharge was also shown to increase the mitochondrial membrane potential, which regulated the fission of mitochondrial membrane and $\mathrm{Ca}^{2+}$ release to the cytosol. ${ }^{97}$ Whether cholesterol processing and mitochondrial modifications differ following efferocytosis or phagocytosis has not been reported, but this aspect has been studied for glucose metabolism. Comparing the impact of apoptotic cell uptake and antibody-mediated phagocytosis, Morioka et al observed that efferocytosis induced aerobic glycolysis, facilitated by higher glucose uptake through GLUT1 upregulation. ${ }^{98}$ This higher glucose consumption by TAM is potentially also immunosuppressive, as a glucose-depleted TME did not support efficient antitumor T-cell functionality ${ }^{99}$ Finally, aerobic glycolysis increases the release of its by-product lactate, which was shown to contribute to the immunosuppressive TME by promoting protumor M2-like TAM polarization, ${ }^{100}$ inhibiting effector $\mathrm{T}$ cells and promoting Treg. ${ }^{99}$ In conclusion, metabolic modifications induced by the cytosolic surcharge after cell engulfment can profoundly impact TAM functions and lead to modifications of the TME.

\section{CONCLUSION AND PERSPECTIVES}

Taking into account many years of in vivo and in vitro experimentation on TAM and their phagocytic function, multiple outcomes are conceivable in the TME. When TAM are appropriately targeted (eg, with antiCD47 antibodies) they do have the capacity to impact on tumor progression, and can collaborate with $\mathrm{T}$ cells (when present in the TME) if their constitutively weak cross-presentation capacities can be reinforced. However, currently used cancer treatments such as radiochemotherapy furnish abundant apoptotic tumor cells that may stimulate predominantly anti-inflammatory and protumor properties of TAM through efferocytosis.

Promoting TAM cross-presentation can have multiple consequences for cancer immunotherapy. Indeed, TAM abundance in solid tumors could facilitate a comprehensive sampling of the whole tumor mass. As tumor heterogeneity, with the presence of antigenically distinct subclones, has been recognized as a limiting factor for an effective T-cell response, ${ }^{101}$ TAM sampling of multiple tumor regions could broaden the repertoire of $\mathrm{T}$ cells by presenting a wide range of tumor antigens more representative of the tumor diversity. In this context, whether TAM have the ability to prime naive $T$ cells against new antigens or whether they can only reactivate $\mathrm{T}$ cells will be an important point to clarify. In line with this, the identified underlying causes of weak cross-presentation by TAM (high proteolysis of antigens, limited translocation of antigens from the phagosome to the cytosol and inhibition of cGAS/STING by TREX1), provide targets to modulate their APC functions and thereby promote
T-cell reactivation or priming. Finally, the fate of cancer cells (apoptotic vs necrotic) after treatment and its impact on TAM functionality has to be better elucidated in vivo, for example, in the context of radio-chemotherapy. This should help clinical trial design, particularly in the case of combining TAM modulation with radio-chemotherapy.

To date, early-phase clinical trials have started to investigate anti-CD47 potentiation of TAM function in patients, with encouraging results for refractory B-cell non-Hodgkin's lymphoma, in which complete responses were observed in $36 \%$ of patients. ${ }^{102}$ Future randomized controlled trials in lymphoma and other type of cancers are eagerly awaited. In the future, combinations of antiCD47 and efferocytosis inhibitors such as those targeting MerTK, and the manipulation of TAM function through modulation of the phagolysosome $\mathrm{pH}$, or the cGASSTING pathway should be exploited in order to unleash the full potential of TAM phagocytic activity. These advances in fundamental immunology and encouraging preclinical results now provide the perspective for innovative clinical trials.

Acknowledgements Figures were created with BioRender.

Contributors ML and PRW designed and wrote the review article. VD contributed to editing the manuscript.

Funding Funding was provided by Fonds Lionel Perrier and Association Frédéric Fellay.

Competing interests None declared.

Patient consent for publication Not required.

Provenance and peer review Not commissioned; externally peer reviewed.

Open access This is an open access article distributed in accordance with the Creative Commons Attribution Non Commercial (CC BY-NC 4.0) license, which permits others to distribute, remix, adapt, build upon this work non-commercially, and license their derivative works on different terms, provided the original work is properly cited, appropriate credit is given, any changes made indicated, and the use is non-commercial. See http://creativecommons.org/licenses/by-nc/4.0/.

\section{REFERENCES}

1 Gentles AJ, Newman AM, Liu CL, et al. The prognostic landscape of genes and infiltrating immune cells across human cancers. Nat Med 2015;21:938-45.

2 Cassetta L, Pollard JW. Targeting macrophages: therapeutic approaches in cancer. Nat Rev Drug Discov 2018;17:887-904.

3 Beltraminelli T, De Palma M. Biology and therapeutic targeting of tumour-associated macrophages. J Pathol 2020;250:573-92.

4 DeNardo DG, Ruffell B. Macrophages as regulators of tumour immunity and immunotherapy. Nat Rev Immunol 2019;19:369-82.

5 Mantovani A, Marchesi F, Malesci A, et al. Tumour-Associated macrophages as treatment targets in oncology. Nat Rev Clin Oncol 2017;14:399-416.

6 Kielbassa K, Vegna S, Ramirez C, et al. Understanding the origin and diversity of macrophages to tailor their targeting in solid cancers. Front Immunol 2019;10:2215.

7 Biswas SK. Metabolic reprogramming of immune cells in cancer progression. Immunity 2015;43:435-49.

8 Fridman WH, Zitvogel L, Sautès-Fridman C, et al. The immune contexture in cancer prognosis and treatment. Nat Rev Clin Oncol 2017;14:717-34.

9 Engblom C, Pfirschke C, Pittet MJ. The role of myeloid cells in cancer therapies. Nat Rev Cancer 2016;16:447-62.

10 Eisenbarth SC. Dendritic cell subsets in T cell programming: location dictates function. Nat Rev Immunol 2019;19:89-103.

11 Garris CS, Arlauckas SP, Kohler RH, et al. Successful anti-PD-1 cancer immunotherapy requires $\mathrm{T}$ Cell-Dendritic cell crosstalk involving the cytokines IFN- $\gamma$ and IL-12. Immunity 2018;49:1148-61. 
12 Hildner K, Edelson BT, Purtha WE, et al. Batf3 Deficiency Reveals a Critical Role for CD8 + Dendritic Cells in Cytotoxic T Cell Immunity. Science 2008;322:1097-100

13 Joffre OP, Segura E, Savina A, et al. Cross-Presentation by dendritic cells. Nat Rev Immunol 2012;12:557-69.

14 Veglia F, Perego M, Gabrilovich D. Myeloid-Derived suppressor cells coming of age. Nat Immunol 2018;19:108-19.

15 Sheng J, Chen Q, Soncin I, et al. A discrete subset of monocytederived cells among typical conventional type 2 dendritic cells can efficiently Cross-Present. Cell Rep 2017;21:1203-14.

16 Helft J, Böttcher J, Chakravarty P, et al. Gm-Csf mouse bone marrow cultures comprise a heterogeneous population of CD11 c+MHCll+ macrophages and dendritic cells. Immunity 2015;42:1197-211.

17 Gautier EL, Shay T, Miller J, et al. Gene-Expression profiles and transcriptional regulatory pathways that underlie the identity and diversity of mouse tissue macrophages. Nat Immunol 2012;13:1118-28.

18 Ruffell B, Au A, Rugo HS, et al. Leukocyte composition of human breast cancer. Proc Natl Acad Sci U S A 2012;109:2796-801.

19 Jaumouillé V, Grinstein S. Receptor mobility, the cytoskeleton, and particle binding during phagocytosis. Curr Opin Cell Biol 2011;23:22-9.

20 Gordon S. Phagocytosis: an immunobiologic process. Immunity 2016:44:463-75.

21 Feng M, Jiang W, Kim BYS, et al. Phagocytosis checkpoints as new targets for cancer immunotherapy. Nat Rev Cancer 2019;19:568-86.

22 Veillette A, Chen J. SIRP $\alpha-C D 47$ immune checkpoint blockade in anticancer therapy. Trends Immunol 2018;39:173-84.

23 Gholamin S, Mitra SS, Feroze AH, et al. Disrupting the CD47SIRP $\alpha$ anti-phagocytic axis by a humanized anti-CD47 antibody is an efficacious treatment for malignant pediatric brain tumors. Sci Transl Med 2017;9:eaaf2968.

24 Wang Y, Xu Z, Guo S, et al. Intravenous delivery of siRNA targeting CD47 effectively inhibits melanoma tumor growth and lung metastasis. Molecular Therapy 2013;21:1919-29.

25 Ring NG, Herndler-Brandstetter D, Weiskopf K, et al. Anti-SIRP $\alpha$ antibody immunotherapy enhances neutrophil and macrophage antitumor activity. Proc Natl Acad Sci U S A 2017;114:E10578-85

26 Iribarren K, Buque A, Mondragon L, et al. Anticancer effects of anti-CD47 immunotherapy in vivo. Oncoimmunology 2019;8:1550619.

27 Abe T, Tanaka Y, Piao J, et al. Signal regulatory protein alpha blockade potentiates tumoricidal effects of macrophages on gastroenterological neoplastic cells in syngeneic immunocompetent mice. Ann Gastroenterol Surg 2018;2:451-62.

28 Home - ClinicalTrials.gov. Available: https://clinicaltrials.gov/ [Accessed 23 Mar 2020].

29 Zhang M, Hutter G, Kahn SA, et al. Anti-CD47 treatment stimulates phagocytosis of glioblastoma by M1 and M2 polarized macrophages and promotes M1 polarized macrophages in vivo. PLoS One 2016;11:e0153550.

30 Willingham SB, Volkmer J-P, Gentles AJ, et al. The CD47-signal regulatory protein alpha $(\mathrm{SIRPa})$ interaction is a therapeutic target for human solid tumors. Proc Natl Acad Sci U S A 2012;109:6662-7.

31 Weiskopf K, Ring AM, Ho CCM, et al. Engineered SIRP $\alpha$ variants as immunotherapeutic adjuvants to anticancer antibodies. Science 2013;341:88-91.

32 Dheilly E, Moine V, Broyer L, et al. Selective blockade of the ubiquitous checkpoint receptor CD47 is enabled by dual-targeting bispecific antibodies. Mol Ther 2017;25:523-33.

33 Liu M, O'Connor RS, Trefely S, et al. Metabolic rewiring of macrophages by CpG potentiates clearance of cancer cells and overcomes tumor-expressed CD47-mediated 'don't-eat-me' signal. Nat Immunol 2019;20:265-75.

34 Sockolosky JT, Dougan M, Ingram JR, et al. Durable antitumor responses to CD47 blockade require adaptive immune stimulation. Proc Natl Acad Sci U S A 2016;113:E2646-54.

35 Gordon SR, Maute RL, Dulken BW, et al. PD-1 expression by tumour-associated macrophages inhibits phagocytosis and tumour immunity. Nature 2017;545:495-9.

36 Schwartz AL, Nath PR, Allgauer M, et al. Antisense targeting of CD47 enhances human cytotoxic T-cell activity and increases survival of mice bearing B16 melanoma when combined with anti-CTLA4 and tumor irradiation. Cancer Immunol Immunother 2019;68:1805-17.

37 Soto-Pantoja DR, Terabe M, Ghosh A, et al. Cd47 in the tumor microenvironment limits cooperation between antitumor T-cell immunity and radiotherapy. Cancer Res 2014;74:6771-83.
38 Biswas SK, Mantovani A. Macrophage plasticity and interaction with lymphocyte subsets: cancer as a paradigm. Nat Immunol 2010;11:889-96.

39 Unanue ER. Perspective on antigen processing and presentation. Immunol Rev 2002;185:86-102.

40 Huang A, Golumbek P, Ahmadzadeh M, et al. Role of bone marrowderived cells in presenting $\mathrm{MHC}$ class I-restricted tumor antigens. Science 1994;264:961-5

41 Cruz FM, Colbert JD, Merino E, et al. The biology and underlying mechanisms of cross-presentation of exogenous antigens on MHC-I molecules. Annu Rev Immunol 2017;35:149-76.

42 Chen DS, Mellman I. Oncology meets immunology: the CancerImmunity cycle. Immunity 2013;39:1-10.

43 Thompson ED, Enriquez HL, Fu Y-X, et al. Tumor masses support naive $T$ cell infiltration, activation, and differentiation into effectors. $J$ Exp Med 2010;207:1791-804.

44 Engelhardt JJ, Boldajipour B, Beemiller P, et al. Marginating dendritic cells of the tumor microenvironment cross-present tumor antigens and stably engage tumor-specific T cells. Cancer Cell 2012;21:402-17.

45 Engelhard VH, Rodriguez AB, Mauldin IS, et al. Immune cell infiltration and tertiary lymphoid structures as determinants of antitumor immunity. J.i. 2018;200:432-42.

46 Calzascia T, Di Berardino-Besson W, Wilmotte R, et al. Cutting edge: cross-presentation as a mechanism for efficient recruitment of tumor-specific CTL to the brain. J Immunol 2003;171:2187-91.

47 Schmidt K, Keller C, Kühl AA, et al. ERAP1-Dependent antigen cross-presentation determines efficacy of adoptive T-cell therapy in mice. Cancer Res 2018;78:3243-54.

48 Amsen D, van Gisbergen KPJM, Hombrink P, et al. Tissue-Resident memory $T$ cells at the center of immunity to solid tumors. Nat Immunol 2018;19:538-46.

49 Malo CS, Huggins MA, Goddery EN, et al. Non-Equivalent antigen presenting capabilities of dendritic cells and macrophages in generating brain-infiltrating CD8 + T cell responses. Nat Commun 2018:9:633

$50 \mathrm{Xu} \mathrm{MM,} \mathrm{Pu} \mathrm{Y,} \mathrm{Han} \mathrm{D,} \mathrm{et} \mathrm{al.} \mathrm{Dendritic} \mathrm{cells} \mathrm{but} \mathrm{not} \mathrm{macrophages}$ sense tumor mitochondrial DNA for Cross-priming through signal regulatory protein $\alpha$ signaling. Immunity 2017;47:363-73.

51 Panni RZ, Herndon JM, Zuo C, et al. Agonism of CD11b reprograms innate immunity to sensitize pancreatic cancer to immunotherapies. Sci Trans/ Med 2019;11:eaau9240.

52 Tang-Huau T-L, Gueguen P, Goudot C, et al. Human in vivogenerated monocyte-derived dendritic cells and macrophages cross-present antigens through a vacuolar pathway. Nat Commun 2018;9:2570.

53 Tseng D, Volkmer J-P, Willingham SB, et al. Anti-CD47 antibodymediated phagocytosis of cancer by macrophages primes an effective antitumor T-cell response. Proc Natl Acad Sci U S A 2013;110:11103-8.

54 von Roemeling CA, Wang Y, Qie Y, et al. Therapeutic modulation of phagocytosis in glioblastoma can activate both innate and adaptive antitumour immunity. Nat Commun 2020;11:1508.

55 Barrio MM, Abes R, Colombo M, et al. Human macrophages and dendritic cells can equally present MART-1 antigen to CD8+ T cells after phagocytosis of gamma-irradiated melanoma cells. PLoS One 2012:7:e40311.

56 Muraoka D, Seo N, Hayashi T, et al. Antigen delivery targeted to tumor-associated macrophages overcomes tumor immune resistance. J Clin Invest 2019;129:1278-94.

57 Klichinsky M, Ruella M, Shestova O, et al. Human chimeric antigen receptor macrophages for cancer immunotherapy. Nat Biotechnol (Published Online First: 23 March 2020).

58 Rybicka JM, Balce DR, Khan MF, et al. Nadph oxidase activity controls phagosomal proteolysis in macrophages through modulation of the lumenal redox environment of phagosomes. Proc Natl Acad Sci U S A 2010;107:10496-501.

59 Delamarre Let al. Differential lysosomal proteolysis in antigenpresenting cells determines antigen fate. Science 2005;307:1630-4

60 Spel L, Boelens J-J, Nierkens S, et al. Antitumor immune responses mediated by dendritic cells: how signals derived from dying cancer cells drive antigen cross-presentation. Oncoimmunology 2013;2:e26403.

61 Colbert JD, Cruz FM, Rock KL. Cross-Presentation of exogenous antigens on MHC I molecules. Curr Opin Immunol 2020;64:1-8.

62 Ackerman AL, Giodini A, Cresswell P. A role for the endoplasmic reticulum protein retrotranslocation machinery during crosspresentation by dendritic cells. Immunity 2006;25:607-17.

63 Giodini A, Cresswell P. Hsp90-Mediated cytosolic refolding of exogenous proteins internalized by dendritic cells. Embo $\mathrm{J}$ 2008;27:201-11. 
64 Rodriguez A, Regnault A, Kleijmeer M, et al. Selective transport of internalized antigens to the cytosol for MHC class I presentation in dendritic cells. Nat Cell Biol 1999;1:362-8.

65 Kovacsovics-Bankowski M, Rock K. A phagosome-to-cytosol pathway for exogenous antigens presented on MHC class I molecules. Science 1995;267:243-6.

66 Houde M, Bertholet S, Gagnon E, et al. Phagosomes are competent organelles for antigen cross-presentation. Nature 2003;425:402-6.

67 Firat E, Saveanu L, Aichele P, et al. The role of endoplasmic reticulum-associated aminopeptidase 1 in immunity to infection and in cross-presentation. J Immunol 2007;178:2241-8.

68 Saveanu L, Carroll O, Weimershaus M, et al. Irap identifies an endosomal compartment required for $\mathrm{MHC}$ class I crosspresentation. Science 2009;325:213-7.

69 Shen L, Sigal LJ, Boes M, et al. Important role of cathepsin $\mathrm{S}$ in generating peptides for TAP-independent MHC class I crosspresentation in vivo. Immunity 2004;21:155-65.

$70 \mathrm{Wu}$ J, Chen ZJ. Innate immune sensing and signaling of cytosolic nucleic acids. Annu Rev Immunol 2014;32:461-88.

71 Ahn J, Xia T, Rabasa Capote A, et al. Extrinsic PhagocyteDependent sting signaling dictates the immunogenicity of dying cells. Cancer Cell 2018;33:862-73.

72 Lorenzi S, Mattei F, Sistigu A, et al. Type I IFNs control antigen retention and survival of $\operatorname{CD} 8 \alpha(+)$ dendritic cells after uptake of tumor apoptotic cells leading to cross-priming. J Immunol Baltim Md 2011;1950:5142-50.

73 Woo S-R, Fuertes MB, Corrales L, et al. Sting-Dependent cytosolic DNA sensing mediates innate immune recognition of immunogenic tumors. Immunity 2014;41:830-42.

74 Vanpouille-Box C, Alard A, Aryankalayil MJ, et al. Dna exonuclease TREX1 regulates radiotherapy-induced tumour immunogenicity. Nat Commun 2017;8:15618.

75 Pereira-Lopes S, Celhar T, Sans-Fons G, et al. The exonuclease TREX1 restrains macrophage proinflammatory activation. J.i. 2013;191:6128-35.

76 Barry KC, Hsu J, Broz ML, et al. A natural killer-dendritic cell axis defines checkpoint therapy-responsive tumor microenvironments. Nat Med 2018;24:1178-91.

77 Goc J, Germain C, Vo-Bourgais TKD, et al. Dendritic cells in tumorassociated tertiary lymphoid structures signal a Th1 cytotoxic immune contexture and license the positive prognostic value of infiltrating CD8+ T cells. Cancer Res 2014;74:705-15.

78 Quail DF, Joyce JA. The microenvironmental landscape of brain tumors. Cancer Cell 2017;31:326-41.

79 Wei J, Chen P, Gupta P, et al. Immune biology of gliomaassociated macrophages and microglia: functional and therapeutic implications. Neuro-Oncol 2020;22:180-94.

80 Lemke G. How macrophages deal with death. Nat Rev Immunol 2019;19:539-49.

81 Rodriguez-Ruiz ME, Vitale I, Harrington KJ, et al. Immunological impact of cell death signaling driven by radiation on the tumor microenvironment. Nat Immunol 2020;21:120-34.

82 Doran AC, Yurdagul A, Tabas I. Efferocytosis in health and disease. Nat Rev Immunol 2020;20:254-67.

83 Filardy AA, Pires DR, Nunes MP, et al. Proinflammatory clearance of apoptotic neutrophils induces an IL-12(low)IL-10(high) regulatory phenotype in macrophages. J Immunol Baltim Md 2010;1950:2044-50.

84 Fadok VA, Bratton DL, Konowal A, et al. Macrophages that have ingested apoptotic cells in vitro inhibit proinflammatory cytokine production through autocrine/paracrine mechanisms involving TGFbeta, PGE2, and PAF. J Clin Invest 1998;101:890-8.

85 Elliott MR, Koster KM, Murphy PS. Efferocytosis signaling in the regulation of macrophage inflammatory responses. J.i. 2017;198:1387-94.
86 Rothlin CV, Ghosh S, Zuniga El, et al. Tam receptors are pleiotropic inhibitors of the innate immune response. Cell 2007;131:1124-36.

87 Kleinclauss F, Perruche S, Masson E, et al. Intravenous apoptotic spleen cell infusion induces a TGF- $\beta$-dependent regulatory T-cell expansion. Cell Death Differ 2006;13:41-52.

88 Yin C, Kim Y, Argintaru D, et al. Rab17 mediates differential antigen sorting following efferocytosis and phagocytosis. Cell Death Dis 2016;7:e2529.

89 Albert ML, Sauter B, Bhardwaj N. Dendritic cells acquire antigen from apoptotic cells and induce class I-restricted CTLs. Nature 1998;392:86-9.

90 Erwig L-P, McPhilips KA, Wynes MW, et al. Differential regulation of phagosome maturation in macrophages and dendritic cells mediated by Rho GTPases and ezrin-radixin-moesin (ERM) proteins. Proc Natl Acad Sci U S A 2006;103:12825-30.

91 Canton J, Khezri R, Glogauer M, et al. Contrasting phagosome pH regulation and maturation in human $\mathrm{M} 1$ and $\mathrm{M} 2$ macrophages. $\mathrm{Mol}$ Biol Cell 2014;25:3330-41.

92 Jung M, Sabat R, Krätzschmar J, et al. Expression profiling of IL-10-regulated genes in human monocytes and peripheral blood mononuclear cells from psoriatic patients during IL-10 therapy. Eur J Immunol 2004;34:481-93.

93 Zagórska A, Través PG, Lew ED, et al. Diversification of TAM receptor tyrosine kinase function. Nat Immunol 2014;15:920-8.

94 Zhou Y, Fei M, Zhang G, et al. Blockade of the phagocytic receptor MERTK on tumor-associated macrophages enhances P2X7RDependent sting activation by tumor-derived cGAMP. Immunity 2020;52:357-73.

95 Vitale I, Manic G, Coussens LM, et al. Macrophages and metabolism in the tumor microenvironment. Cell Metab 2019;30:36-50.

96 Cui D, Thorp E, Li Y, et al. Pivotal advance: macrophages become resistant to cholesterol-induced death after phagocytosis of apoptotic cells. J Leukoc Biol 2007;82:1040-50.

97 Wang Y, Subramanian M, Yurdagul A, et al. Mitochondrial fission promotes the continued clearance of apoptotic cells by macrophages. Cell 2017;171:331-45.

98 Morioka S, Perry JSA, Raymond MH, et al. Efferocytosis induces a novel SLC program to promote glucose uptake and lactate release. Nature 2018;563:714-8.

99 Thommen DS, Schumacher TN. T cell dysfunction in cancer. Cancer Cell 2018;33:547-62.

100 Colegio OR, Chu N-Q, Szabo AL, et al. Functional polarization of tumour-associated macrophages by tumour-derived lactic acid. Nature 2014;513:559-63.

101 McGranahan N, Furness AJS, Rosenthal R, et al. Clonal neoantigens elicit $\mathrm{T}$ cell immunoreactivity and sensitivity to immune checkpoint blockade. Science 2016;351:1463-9.

102 Advani R, Flinn I, Popplewell L, et al. Cd47 blockade by Hu5F9-G4 and rituximab in non-Hodgkin's lymphoma. $N$ Engl $\mathrm{J}$ Med 2018;379:1711-21.

103 Bruhns P. Properties of mouse and human IgG receptors and their contribution to disease models. Blood 2012;119:5640-9.

104 Chao MP, Jaiswal S, Weissman-Tsukamoto R, et al. Calreticulin is the dominant pro-phagocytic signal on multiple human cancers and is counterbalanced by CD47. Sci Trans/ Med 2010;2:63ra94.

105 Chen J, Zhong M-C, Guo H, et al. Slamf7 is critical for phagocytosis of haematopoietic tumour cells via Mac-1 integrin. Nature 2017;544:493-7.

106 Barkal AA, Weiskopf K, Kao KS, et al. Engagement of MHC class I by the inhibitory receptor LILRB1 suppresses macrophages and is a target of cancer immunotherapy. Nat Immunol 2018;19:76-84. 